\title{
FRONTERAS Y REDES TRANSFRONTERIZAS: UNA NUEVA ORGANIZACIÓN DEL TERRITORIO EN LA ARGENTINA DEL MERCOSUR
}

La Autora es docente de la Universidad Nacional de la Plata y de la Universidad de Buenos Aires.(Argentina) e Investigadora Principal del CONICET.

\begin{abstract}
RESUMEN. EI trabajo trata de comprender las transformaciones territoriales emergentes de la evolución del modelo económico-político neoliberal en el período reciente, a partir de una revisión de las principales características de este proceso en las regiones centrales del planeta y en el cono sur de América Latina. Al respecto el artículo explora el sentido y la dinámica de las nuevas obras públicas y de las actividades económicas con la finalidad de describir los efectos territoriales de estos procesos en marchas, destacando la importancia que desempeñan dentro de los mismos las grandes obras de transporte y energía, como así también los efectos sociales de las transformaciones territoriales emergentes y las limitaciones de rol tradicional del Estado-Nación para hacer frente a los cambios.
\end{abstract}

ABSTRACT. FRONTAIRES AND TRANS-FRONTAIRES NETS: MERCOSUR and the new Argentina' territorial organisation.

This article tries to comprehend territorial transformations as a result of neo-liberal schemes developed recently. It reviews the main characteristics of that process looking at certain regions of the world and particularly at the South cone of Latin America. It explores the sense and dynamic of new built infrastructure and economic activities with the aim to describe changes generated as a result of the process. It highlights the important role played by main transport and energy infrastructures recently built. Also, discusses the social consequences in the context of severe constraints that face the old fashion nation-state in dealing with these new scenarios.

\footnotetext{
"...lo que aún es invisible a los ojos pero no por ello menos esencial" (Saint Exupery)

"Contribuer à penser, en géographie, cette recomposition du monde qui s'opère sous nos yeux" (Foucher, 1991)

"En la perspectiva de una ordenación transnacional del territorio, cualesquiera que sean sus agentes y las competencias y atribuciones de que dispongan, es necesario asimilar primero las culturas de los territorios y su enfoque de ordenación, así como movilizar y organizar a los operadores en torno a temas y proyectos articuladores, antes de abordar operaciones de envergadura" (Europa 2000+, Comunidad Europea, 1994)
} 


\section{INTRODUCCIÓN}

El trabajo que se presenta a consideración resume reflexiones y conclusiones de una línea de investigación que trata de comprender las transformaciones territoriales emergentes de la evolución del modelo económico-político neoliberal en el período reciente.

Desde el escenario mundial a los regionales y locales, en general, éstas transformaciones incluyen imágenes comunes pero diferentes: fragmentación, centralización, concentración, segregación, etc. Las que describen las características que adquiere el proceso reciente de disputa de los territorios como consecuencia de los procesos de globalización de las economías y de integración de las regiones.

Dos sectores -por su funcionalidad al modelo económico adoptado- resultan ser los atendidos preferentemente por el Estado y el capital privado: la energía y el transporte.

En este marco, los territorios localizados en las fronteras, tanto internas como externas de los mercados, ${ }^{[1]}$ registran los mayores efectos en las actividades económicas, en la utilización, ocupación y apropiación del suelo, en la dotación de infraestructuras de transporte y energía y en los ambientes en general. En ellos se registran cambios significativos en la dinámica poblacional, en la calidad de vida de diferentes sectores sociales, en la aplicación de nuevas modalidades de gestión y en la articulación entre políticas locales, regionales, nacionales y supranacionales a ambos lados de las fronteras.

En el estudio se acepta el imperativo de reflexionar sobre cómo diseñar nuevos modelos capaces de intentar una necesaria recomposición en la organización de estos territorios fronterizos. A partir de describir aquellos escenarios en los que se manifiesta la heterogeneidad de la nueva pobreza, los fenómenos de exclusión más allá de lo económico, la ausencia de gasto social, en general la modernización excluyente. Contrastando con la materialización de redes transfronterizas y estrategias de vinculación biocéanicaslos Nuevos Grandes Proyectos ${ }^{[2]}$ que caracterizan y definen este inicio de siglo.

La reflexión y el análisis sobre estos territorios y sus proyectos asociados, obliga para su comprensión profundizar la investigación en aspectos como:

n Las infraestructuras facilitadoras de los intercambios nacionales, internacionales, fronterizos y, especialmente, la realización de infraestructuras de transporte, tanto viales como ferroviarias, que materialicen corredores que vinculen los océanos Atlántico y Pacífico y la interconexión de los sistemas energéticos nacionales que refuerzan la continuidad física de los territorios y contribuya a la integración industrial.

n La evolución y los impactos de los flujos de intercambio entre territorios en vías de integración: la apertura económica, el transporte de mercaderías, la oferta de servicios, sus impactos sobre las economías transfronterizas, la localización de nuevas inversiones, nuevas actividades, la evolución de las actividades preexistentes.

n La movilidad de las personas y los procesos migratorios que caracterizan estas nuevas regiones fronterizas (fronteras entre mercados, fronteras internas, fronteras internacionales).

n La emergencia de nuevos actores y nuevas prácticas sociales, el reposicionamiento de actores preexistentes, públicos y privados y en los diferentes niveles y a sus nuevas lógicas de articulación, los nuevos instrumentos, mecanismos y modalidades de gestión de estos territorios, con sus aspectos positivos y negativos: regulación y desregulación, control, armonización de políticas, competencia, etc.

Entre otros aspectos conceptuales se profundizó e incorporó el concepto de redes en la teoría que a partir de los años 80 se introduce en las relaciones internacionales. En geografía, así como en antropología y en sociología, la noción de redes llena lagunas que aparecen en la confrontación de los viejos modelos teóricos con la realidad actual de transformaciones económicas y reestructuración de los territorios. 


\section{LA PROBLe MÁtICA de LOS ESTADOS FRENTE AL PROCESO DE REESTRUCTURACIÓN ECONÓMICA}

El proceso de reestructuración económica está hegemonizado por sus actores y agentes más dinámicos, fundamentalmente las empresas transnacionales (cuyas sedes se localizan en las principales ciudades de los países centrales) y sólo son en ellas que los Estados reúnen las condiciones para mantener políticas sociales en mayor o menor medida efectivas y atenuar los efectos negativos de la reestructuración.

Las corporaciones multinacionales procuran mejorar su posicionamiento en los mercados mundiales, creando nuevas condiciones para el aprovechamiento de recursos y nuevas pautas de localización, como una manera de optimizar en una escala mundial las modalidades de producción, tendientes a la disminución de costos. Estas condiciones son generadas en teoría -al menos en los discursos oficiales-, por los avances en las telecomunicaciones y la informatización, que posibilitan obras de infraestructura de transporte y energía y facilitan la liberalización del comercio internacional, pero, citando a Ernst Mandel, debemos tener en cuenta también que: "La internacionalización de las fuerzas productivas tiende a la globalización de viejos y nuevos problemas, es decir a la imposibilidad de resolverlos en la escala nacional e incluso continental, como los del subdesarrollo: el hambre, las epidemias, la guerra, los que se suman a los nuevos problemas, como las amenazas de guerra nuclear y los desastres ambientales". Mientras que el Estado es incapaz de resolver los problemas citados, el sistema no permanece pasivo, reacciona "con sentido de autodefensa", elaborando semisoluciones o transformaciones parciales. Frente a la impotencia del Estado-nación, el gran capital se orienta a la creación de instrumentos supranacionales. (Mandel, 1993).

Los acuerdos binacionales, las asociaciones supranacionales regionales y continentales, como la Comunidad Económica Europea; los mecanismos de coordinación económica internacional, enmarcados en los procesos más generales de globalización, como los encuentros anuales del Grupo de los 7+1; o el reforzamiento del papel de ciertos organismos mundiales, como la ONU, el FMI, el Banco Mundial, la Organización Mundial de Comercio, etc. (Oman, 1994) y hasta una OTAN, reforzada por la incorporación de estados que antes formaban parte del bloque socialista, dan cuenta de este planteamiento. También en esta misma línea se desarrolla el rol de gendarme del mundo por parte de los Estados Unidos y la creciente resistencia -lejana de la competencia- por parte de la Unión Europea, Rusia o China.

Los procesos de internacionalización y de integración de las regiones en los países de América Latina, requieren para su comprensión estudiar los impactos territoriales combinados de la relación entre integración comercial externa y modernización neoliberal interna (Ramírez y Pradilla Cobos, 1993). Entre sus efectos se destacan la desindustrialización, la aceleración desigual de la urbanización con niveles cada vez mayores de segregación y de exclusión social, la aparición de nuevos enclaves, nuevos desiertos interiores, etc.

\section{LA DISPUTA DE TERRITORIOS HACIA SU INSERCIÓN EN EL ESCENARIO MUNDIAL}

A medida que las políticas de integración se consolidan, se comienza a hacer evidente una nueva lógica territorial dentro de la que se identifican diferentes grados de compromiso territorial en y desde Argentina:

a) El rol hegemónico que cobran ciertas metrópolis (São Paulo, Buenos Aires), concentrando el poder de decisión económica, política y financiera. En el contexto de una economía cada vez más "relacional", la calidad de la relación entre los actores económicos es un aspecto crítico. La vinculación entre los distintos actores de la cadena productiva se ve facilitada por la geografía densa del medio urbano y la metrópolis actúa como un gran intercambiador que permite el arreglo flexible de las cadenas productivas.

Frente a esta situación, los actores hegemónicos y algunos no tanto, públicos y privados de otras ciudades, capitales nacionales como Montevideo, Santiago de Chile, Asunción, de estados y 
provincias, como Curitiba, Porto Alegre, Rosario, Córdoba, Mendoza y otros grandes centros buscan mejorar sus condiciones de inserción. ${ }^{[3]}$

b) La transformación y/o adaptación de los circuitos y redes de producción-distribución que determinan territorios que se verán beneficiados por efectos favorables y otros que se verán impactados de manera desfavorable.

c) Una serie de proyectos u obras de infraestructura, los Nuevos Grandes Proyectos, de producción y transporte de energía, de comunicaciones y de transporte de cargas y pasajeros (puentes, túneles, autopistas) que materializan en el espacio los crecientes flujos comerciales de los mercados ampliados creando redes transfronterizas.

d) La profunda transformación de las áreas fronterizas que, más allá de su tradicional carácter contradictorio de áreas que marcan las diferencias entre países a la vez que reconocen una lógica propia que las identifica, son hoy áreas estratégicas en las que se concentran los efectos del despliegue de la reestructuración económica.

En este marco, los territorios localizados en las fronteras, tanto internas como externas de los mercados, ${ }^{[4]}$ registran los mayores efectos en las actividades económicas, en la utilización, ocupación y apropiación del suelo, en la infraestructura de transporte y energía y en los ambientes en general. En ellos se registran cambios significativos en la dinámica poblacional, en la calidad de vida de diferentes sectores sociales, en la aplicación de nuevas modalidades de gestión y en la articulación entre políticas locales, regionales, nacionales y supranacionales a ambos lados de las fronteras.

De los procesos citados resultarían regiones que obtienen beneficios y regiones que serán excluidas. En las primeras resultarían beneficiados los sectores más poderosos, los grandes capitales monopólicos y los sectores medios integrados; en las segundas, los efectos negativos se difundirán en todos los sectores sociales.

En los últimos años las dinámicas asociadas a los procesos de reestructuración económica como los procesos de integración en el Cono Sur fueron dando origen al desarrollo de nuevos grandes proyectos de infraestructura de transporte y energía. Estos proyectos, juntamente a las nuevas modalidades de gestión territorial, están dando lugar a un nuevo mapa de la región y a modificaciones en el rol de las regiones fronterizas. Obedeciendo a las necesidades de los mercados de la Unión Europea y el Sudeste Asiático y ante las dificultades del cruce del Canal de Panamá se encontrarían en desarrollo proyectos de corredores de transporte que se inscribirían en la estrategia de vinculación biocéanica Atlántico-Pacífico cruzando América del Sur (Laurelli y Schweitzer, 1995).

A lo largo del período reciente se comienzan a manifestar los elementos de una nueva configuración territorial, un nuevo mapa de la subregión. Desde los objetivos del proceso integrador en esta etapa reciente, se puede suponer que los efectos tendrán correlato en alteraciones a nivel de circuitos de producción-distribución-consumo, en su expansión en el ámbito de nuevos mercados nacionales e internacionales y su repercusión a nivel regional y local. Estos efectos se producirán alterando su dinámica y/o su propia estructura -en aquellos que ya existen en regiones dentro de su país, o comparten varios países vecinos o son parte de un circuito transnacional- produciendo consecuencias que van de un desarrollo expansivo a su reconversión y/o desaparición.

Este proceso se ve acompañado de nuevos requerimientos de infraestructuras regionales y locales, tanto de comunicaciones y transporte como de energía, con iniciativas para la construcción de nuevas grandes obras.

En los territorios de los países del sur, en los que se aplican políticas económicas y sociales cada vez más excluyentes, los únicos puntos fijos son los que se constituyan como articulaciones del transporte de cargas, mientras que los vínculos serán las líneas por donde pasarán los flujos entre los distintos centros integrados a los mercados mundiales. Estas articulaciones, ya que para la vinculación entre centros existen varias alternativas de medios de transporte y trazado, serán los puertos, puentes y túneles.

Como toda política que implica la posible expectativa de generación de empleo, se visualiza la presencia incipiente de fenómenos migratorios -los que se puede suponer aumentarán en la medida 
en que comience a cristalizar la nueva dinámica territorial- de muy diversas características acrecentados por los efectos de las políticas de ajuste. ${ }^{[5]}$

El rol que asume el Estado en el marco de la reformulación de sus funciones y la disminución de su capacidad de regulación incide sobre la modalidad de gestión de las obras de infraestructura, en la inserción del sector privado en ellas y en la disposición de mecanismos destinados a la satisfacción de las demandas sociales que estas implantaciones produzcan, tanto en cuanto al nivel de satisfactores urbanos como de atención a la problemática de empleo, salud, educación, seguridad, ambiental, migraciones, etc.

La presencia de organismos financieros internacionales como el BID y el BIRF-Banco Mundial, de cooperación japoneses y europeos y grupos económicos en general, ha generado avances significativos en el desarrollo de los proyectos, funcionales a un mismo objetivo general que puede trasladarse a distintos tipos de proyectos: responder al modelo económico adoptando una solución de rentabilidad asegurada.

\section{¿Nuevos roles para las fronteras[6] del Cono Sur?}

La mayor parte de las fronteras políticas del Cono Sur se determinan por límites físicos que coinciden con los ríos de la Cuenca del Plata o las altas cimas de la Cordillera de los Andes (en especial Argentina). Por otro lado, gran parte de las líneas de delimitación de las fronteras separan ecosistemas comunes e incluso circuitos económicos y relaciones sociales anteriormente integrados. Las vías navegables, en particular las que forman la Cuenca del Plata, han significado para los países del Cono Sur, el medio por el cual han sido desarrollados los primeros acuerdos y experiencias de integración. Aunque, en general, de manera reducida, con proyectos de diversa escala, desde las grandes represas de Itaipú, Salto Grande y Yaciretá, enclaves de generación de energía hidroeléctrica, hasta la unificación de los controles de frontera, los países de la cuenca vienen intentando en numerosas ocasiones, actuar sobre las poblaciones y actividades de estas regiones fronterizas.

Las regiones fronterizas a lo largo de la historia fueron, con excepción de algunos pocos emprendimientos puntuales, receptoras de la implantación de equipamientos y actividades militares orientados por las "hipótesis de conflicto" cumpliendo el rol marginal de "borde" en un largo período que se extiende desde la independencia hasta fines de la década del '80. Actualmente están siendo los territorios del despliegue más inmediato de los procesos de integración y de la reestructuración económica, tanto por la presencia como por la ausencia de actividades y proyectos relacionados con los mismos procesos.

\section{LAS REGIONES FRONTERIZAS}

Las fronteras se caracterizan por la existencia de diferencias de importancia variable, según el caso, entre dicha área y el resto de cada uno de los dos países, desde el punto de vista de los efectos de la presencia de la frontera. No puede definirse un límite a partir del cual dejan de sentirse los efectos, la frontera es un área de interfase, dado que en general las influencias recíprocas determinan especificidades de comportamiento de dicha área en relación con el resto de los respectivos países.

En los casos de realización de grandes proyectos de infraestructura - de transportes, generación de energía de nuevas actividades productivas, grandes equipamientos etc.- las áreas fronterizas y en general los territorios que reciban las nuevas localizaciones podrán verse insertas más directamente en las dinámicas citadas en la forma de procesos de inclusión territorial -lo que no significa inclusión social- mientras que en las áreas restantes se desatarán procesos de sentido inverso, de signos excluyentes y regresivos.

Por otra parte, los instrumentos de políticas de integración que se están poniendo en práctica desde distintos niveles de decisión, -nacionales, provinciales y municipales, binacionales o regionales- en términos de conformación de mercados comunes y de integración física, orientados a la facilitación y agilización del transporte, la integración energética y provisión de servicio, están significando 
modificaciones en las modalidades de gestión de los territorios fronterizos, permitiendo redefinir los roles para las fronteras entre los países de la región:

n Fronteras de mercados y fronteras nacionales,

n Territorios de paso de cargas - corredores de transporte- en el interior de los territorios nacionales, que unen regiones fronterizas alejadas,

n Fronteras que separan ecosistemas, actividades productivas, culturas comunes,

n Iniciativas de integración fronteriza -con mayores niveles de interacción entre sociedades de las dos márgenes- e iniciativas de integración binacional-grandes represas, puentes- localizadas en regiones fronterizas,

n Regiones fronterizas en las que los Estados nacionales mantienen una presencia fuerte, con superposición de jurisdicciones, y fronteras en las que los niveles locales son las que toman la iniciativa de integración,

n Regiones fronterizas fuertemente integradas entre las dos márgenes -con redes y organismos de gestión binacional o trinacional fronteriza-, algunas de ellas débilmente integradas a sus respectivos territorios nacionales, ciudades "par de frontera", 7$]$ algunas sobre frontera seca, otras vinculadas por puentes sobre ríos, con o sin integración de la provisión de servicios públicos e infraestructura por red.

\section{CONSIDERACIONES FINALES}

La sucesión de los proyectos de intervención en obras públicas de infraestructura y la radicación y/o relocalización de actividadesproductivas se está dando tanto en las regiones fronterizas -con mayores intensidades- como en las regiones metropolitanas.

Estos proyectos, por responder al modelo de acumulación actualmente en crisis, provocan modificaciones en la conformación de los territorios, en su uso, ocupación y apropiación, de carácter más o menos inmediato. Pueden sin embargo aparecer situaciones diversas según la forma que sean aprovechada la infraestructura y los equipamientos instalados, generándose eventualmente nuevas áreas periféricas y la incorporación de otras al sistema económico internacional.

Los proyectos, tanto los ya concretados como los que se encuentran en marcha -aun en estos momentos- o apenas en estado de idea o proyecto, están vinculados a la localización de centros productores de bienes exportables, puntos de salida y de entrada de estos productos, centros de transferencia, de servicios de apoyo al transporte, etc. Cuentan con la participación de los Estados nacionales y provinciales y en mucha menor medida locales, pero las modalidades en que en ésta se da se están modificando a favor de una cada vez menor intervención estatal en la construcción del rol de articulador de actores y facilitador de la concreción de los proyectos.

Durante la operación de los emprendimientos, las funciones del Estado son reguladoras, dejando en manos de las empresas concesionarias -a riesgo de los capitales privados- la recuperación de las inversiones realizadas. Esto no implica que el Estado no efectúe gastos, ya que por un lado debe financiar los estudios previos y por otro nunca queda claro las responsabilidades de las empresas sobre la resolución de los efectos negativos de la construcción de los proyectos.

Se deben considerar además las relaciones de subordinación y competencia que en el marco actual se establecen entre los distintos niveles de decisión, que terminan expresándose en nuevas configuraciones territoriales en las que aparecen las citadas regiones que ganan y regiones que pierden. No puede afirmarse que la localización de un proyecto traiga aparejado el desarrollo de la región de implantación por la radicación de nuevas actividades, ya que son numerosas las experiencias de caso que tienden más a la constitución de enclaves que a la difusión de los presuntos beneficios a los sectores locales, y aun menores los que, dentro de estos últimos, se generan condiciones para la mejora de la calidad de vida de la población anterior y los nuevos migrantes que puedan localizarse de manera permanente una vez concluida la obra. ${ }^{[8]}$ 
En el caso de los proyectos en desarrollo en las regiones fronterizas se agregan nuevos factores, como los de ser obras binacionales o de asociaciones entre varios países, que llevan a la necesidad de proyectar nuevas modalidades de gestión. Si además se suman a estas consideraciones las relacionadas con los avances en los procesos de integración de los países en asociaciones supraestatales, la problemática a estudiar se torna en una serie de cuestiones de mayor complejidad que deben analizarse de manera articulada. Asimismo deben considerarse los objetivos enunciados en los distintos momentos de realización y las orientaciones -explicitadas o no- sobre el sentido de la integración que se expresan en los contenidos del proyecto.

Considerando los avances en los procesos de integración en el Cono Sur y las características que estos procesos asumen, por el momento la gestión de los grandes proyectos de infraestructura en marcha -la integración física- se da de manera desligada de las conversaciones y celebración de acuerdos en el marco de la integración económica; mientras que por otro lado los mismos procesos de integración, restringidos a los aspectos comerciales y en menor medida, económicos en general, pueden derivar tanto hacia otros perfiles y niveles de integración más avanzados como retroceder aunque es la probabilidad menos real- hacia los niveles previos a los actuales procesos. Estas dos características corren en forma paralela, dependiendo del desarrollo de nuevas modalidades de gestión la posibilidad de combinarse en una política enmarcada en una estrategia y proyectos generales (Schweitzer, 2000).

Por otra parte entran en juego además los rumbos que tomen los procesos a nivel mundial que pueden derivar tanto en una mayor articulación y agilización de los intercambios entre los distintos territorios, con escasa dispersión de los beneficios a la asociación de países desarrollados y las nuevas ciudades mundiales; o hacia una regionalización cerrada con bloques protegidos. En los últimos años las tendencias, tal como se afirma en este trabajo, parecen orientarse hacia este último escenario, mientras que por otro lado, ninguno de los dos estaría demostrando ser generador de menores niveles de exclusión social (Arrighi, 1998).

En el marco descrito, las condiciones para la inserción de las regiones metropolitanas en el Cono Sur en el nuevo escenario internacional definido por la mundialización varían según la capacidad de quienes estén a cargo de su gestión para atraer la localización de actividades dinamizadoras de distinto tipo.

Al respecto puede afirmarse que son cada vez más reducidos los desarrollos de iniciativas estatales de planificación regional, tanto a nivel nacional como a niveles menores. La superposición de jurisdicciones, atribuciones y modalidades de vinculación de las poblaciones y gobiernos locales con las distintas escalas de gobierno y con los sectores privados, incluso de países vecinos o de carácter binacional, hace que la aplicación de los criterios citados se articulen en intensidades y tiempos diferenciados, tanto hacia el interior de cada país, como ambos lados de la frontera. Esta situación debilitará la capacidad de los gobiernos locales para hacer frente a los efectos recesivos sobre los sectores más desprotegidos de la población, a las actividades que se desarrollan a nivel local, a los requerimientos sumados por las políticas de integración, y a la canalización de las demandas locales a los niveles superiores de gobierno (Rofman y Simone, 1989).

Estas cuestiones se agudizan en el marco de los procesos de descentralización, desregulación, privatización y transferencia de nuevas atribuciones a los gobiernos locales. Los modelos de gestión local vigentes sufren modificaciones de diversa índole y grado según las características que tomen las articulaciones de los distintos actores y sectores sociales intervinientes en los niveles locales.

De acuerdo a las situaciones territoriales planteadas, si los territorios localizados en las regiones fronterizas son los que estarían recibiendo más directamente las afectaciones de los avances de estos procesos, dentro de la competencia por la atracción de capitales que se da tanto en los niveles nacionales como en los regionales y municipales y teniendo en cuenta además las asimetrías en las dinámicas poblacionales y económicas y en las modalidades de gestión territorial a ambos lados de las fronteras, las regiones metropolitanas que resulten insertas en mejores posiciones son las que cuentan con la ventaja de la localización previa de un conjunto de condiciones con el que no cuentan otras áreas, acumulado a lo largo de la mayor de la historia de nuestros países. 
Aún en las denominadas "ciudades mundiales" en los países del norte, se están acentuando en estos años los fenómenos de segregación social y espacial hacia el interior de las mismas (Sassen, 1998). Estos efectos se darán en forma más agravadas en las que pocas metrópolis consigan entrar en la lista desde el sur del planeta.

A lo largo de la lectura de estudios sobre efectos resultantes de la implantación de grandes proyectos de infraestructura, en particular las relacionadas con el transporte, se pueden enumerar afectaciones de todo tipo, que van desde las modificaciones en el medio físico, natural y construido, pasando por las actividades productivas, los usos y la ocupación del suelo, los efectos sobre las sociedades asentadas en el lugar y los procesos desatados en distintas escalas -constitución de enclaves de diversas características, fenómenos migratorios, satisfacción o no de las necesidades básicas de distintos sectores, mayores o menores niveles de exclusión social, etc.

En los niveles sub-regionales y locales, en relación a los nuevos criterios de localización y actividades productivas y las infraestructuras necesarias para su inserción, marcados por el modelo neo-liberal, se puede sostener que los sectores más integrados y transnacionalizados son los que estarían contando con los recursos necesarios, lo que les permitiría estar en condiciones de llevar adelante las obras de infraestructura y servicios que requieran (Arantes, Vainer y Maricato, 2000). Esto se dará sean o no proporcionadas por el Estado, bajo distintas formas de concesión, privatización, etc.

Ante la debilidad impuesta a los estados, mayor aún en sus niveles más locales, se verá comprometida la provisión de obras y servicios desde el sector público hacia actividades productivas y sectores sociales no ligados a las nuevas condiciones. Como consecuencia de esto se dará todavía en mayor nivel el agravamiento de las condiciones de vida y de la situación social en general en los territorios que no reciban localizaciones de actividades dinamizadoras. Se producirán entonces nuevos desplazamientos de actividades y población desde las áreas cada vez mas excluidas hacia las que puedan llegar a estar cada vez más integradas.

\section{BIBLIOGRAFÍA CITADA Y CONSULTADA}

ARANTES, OTILIA, VAINER, CARLOS Y MARICATO, HERMINIA

2000. A cidade do pensamento único. Desmanchando consensos. Vozes, Petrópolis.

ARRIGHI, GIOVANNI

1998. A ilusão do desenvolvimento, Vozes, Petrópolis.

CASTELLO, IÁRA; OTERO SCHAFFER, NEIVA (ORGANIZADORES)

1994. Fronteira no Mercosul. Porto Alegre: Ed. Da Universidade/UFRGS y Prefeitura Municipal de Uruguaiana. COMISION EUROPEA

1995. (Políticas Regionales de la Comunidad Europea). Europa 2000+. Cooperación para la ordenación del territorio europeo. Oficina de Publicaciones Oficiales de las Comunidades Europeas. Luxemburgo.

FOUCHER, MICHEL

1991. Fronts et frontières, un tour du monde géopolitique, Fayard, Francia,

GONZALES POSSE, Ernesto; BOLOGNESI-DROSDOFF, María C.; ITURRIZA, Jorge E.; VALENCIANO, Eduardo O

1990. La frontera como factor de integración. BID-INTAL, Buenos Aires.

LAURELLI, ELSA

1996. "Integration scenarios: projects, issues and problems in the southern cone", en UNISA. Latin American Report. Pretoria, Sud Africa, volumen 12, n. 2.

1981. Estudios particularizados en Areas Fronterizas sobre ciudades pares de frontera. Un estudio de caso: Eagle Pass-Piedras Negras, México, mimeo.

1986. "El sistema de poder en la producción del espacio urbano-rural. El caso de la microrregión de Río Escondido", en PADUA, Jorge; VANNEPH, Alain. Poder local, poder regional. El Colegio de México/CEMCA. México.

1992. "Reestructuración económica en América Latina: ¿integración o fractura de los territorios fronterizos?”, en lára CASTELLO et al. Fronteiras na America Latina, espaços em transformaçao. Editora da UFRGS, Porto Alegre, Brasil.

LAURELLI, ELSA; MONTAÑA, ELMA; SCHWEITZER, ALEJANDRO; SCHWEITZER, MARIANA 
1995. Los escenarios de la integración: Sociedades excluidas y territorios involucrados en América Latina en su relación con la Cuenca del Pacífico. Presentación en el Vii Congreso de la Federación Internacional sobre Estudios de América Latina y el Caribe, Taipei, Taiwan, Junio,

LAURELLI, ELSA Y ALEJANDRO ROFMAN

1991. Políticas de Integración Subregional: Escenarios socio-territoriales. Hipótesis de partida para el caso argentino. Ponencia presentada en el Seminario Internacional "Integración Latinoamericana y Territorio. Transformaciones socio económicas, políticas y ambientales en el marco de las políticas de ajuste", Instituto de Geografía de la Facultad de Filosofía y Letras de la UBA y el CEUR. Buenos Aires, 3 al 6 de diciembre.

LAURELLI, ELSA. (COORD.); SCHWEITZER, ALEJANDRO; ZAMUNER, LIDIA (COLAB.)

1993. El impacto regionales del puente Buenos Aires-Colonia en el marco de las políticas de integración. MERCOSUR y Tratado de Cooperación con Chile. Realizado en el mes de diciembre de 1993 para la Comisión Binacional del Puente Buenos Aires-Colonia. Buenos Aires.

LAURELLI, ELSA; SCHWEITZER, ALEJANDRO

1995. "Infraestructure or Megastructures? Proposed project may transform South American's Southern Cone", en Land lines. Cambridge, MA: Ed. Lincoln Institute of Land Policy.

LAURELLI, ELSA, ALEJANDRO SCHWEITZER Y ELMA MONTAÑA

1998. "El Despliegue Territorial de la Reestructuración Económica y los Procesos de Integración: Buenos Aires y Mendoza: Dos Metropolis en la disputa por la Hegemonía Regional y la inserción en un contexto de exclusión", en DE MATTOS, Carlos; Daniel HIERNAUX NICOLAS y Darío RESTREPO BOTERO (comp.) Globalización y Territorio. Impactos y perspectivas, Santiago de Chile, Fondo de Cultura Económica, Pontificia Universidad Católica de Chile.

LAURELLI, ELSA (COORD.) CARRIQUIRIBORDE HELENA, LILJESTHRÖM MOIRA, MONTAÑA ELMA, RAPOSO ISABEL, SCHWEITZER ALEJANDRO Y SCHWEITZER MARIANA

2001. "El despliegue territorial de la reestructuración económica: ¿integración regional o desintegración social en América Latina?", en Actas del VI Seminario Internacional de la Red Iberoamericana de Investigaciones sobre Globalización y Territorio, UNR, Rosario.

MANDEL, ERNST

1993. "Globalización, interdependencia y bloques económicos regionales", en El Cielo por asalto, Buenos Aires.

M.M.A.U.V. PCIA. DE MENDOZA-CEUR

junio de 1992 a abril de 1993. Programa de Ordenamiento Territorial del Corredor Andino. Elsa Laurelli (Dirección y Coordinación). Mendoza y Buenos Aires,

OMAN, CHARLES

1994. Globalisation et régionalisation: quels enjeux pour les pays en développement. Etudes du Centre de Développement, OCDE, París.

OTERO SCHAFFER, NEIVA

1994. "Morador e moradia em periferia de cidade de fronteira: condiçôes sócio-económicas em villa de Santana do Livramento", en CICCOLELLA, P.; LAURELLI, E.; ROFMAN, A.; YANEZ, L. Integración latinoamericana y territorio. Transformaciones socio-económicas, políticas y ambientales en el marco de las políticas de ajuste. Ed.: CEUR e Instituto de Geografía de la Facultad de Filosofía y Letras, UBA, Buenos Aires.

PRADEAU, CHRISTIAN

1994. Jeux et enjeux des frontières. Presses Universitaires de Bordeaux, Francia.

PRADILLA COBOS, EMILIO

1996. "México en el Tratado de Libre Comercio de América del Norte: El futuro en busca del presente", en COUFFIGNAL, Georges; De la REZA, Germán (Eds.): Los procesos de integración en América Latina. Enfoques y perspectivas. Institute of Latin American Studies, Stockholm University, Suecia.

RAFFESTIN, CLAUDE

1993. "Autour de la fonction sociale de la frontière", en Espaces et société, n. 70-71, Université de Genève.

RAMIREZ, BLANCA; PRADILLA-COBOS, EMILIO

1993. "El Tratado Norteamericano de Libre Comercio y la integración territorial de México a Estados Unidos", en Revista Interamericana de Planificación. v. XXVI, n. 103, Ed. SIAP, San Antonio, Texas, EUA.

SALAMA, PIERRE

1995. "Amérique latine: intégration sans désintégration?", en Problèmes d' Amérique latine, n. 19, oct-dec. 1995.

La Documentation française. Paris.

SALVIA, AGUSTÍN; PANAIA, MARTA (COMP.)

1997. La Patagonia privatizada. Colección CEA-CBC, Buenos Aires.

SASSEN, SASKIA

1998. As cidades na economia mundial. Studio Nobel, São Paulo,

SCHWEITZER, ALEJANDRO 
2000. Intégration régionale et aménagement du territoire dans le Mercosur. Frontières, réseaux et dynamiques transfrontalières, Tesis de doctorado, Paris, IHEAL.

VELTZ, PIERRE

1996. Mondialisation, Villes et Territoires. L'économique d'archipel. Paris, Presses Universitaires de France. Collection "Economie et liberté".

[1] Se introducen los conceptos de "frontera interna" y "externa" de los mercados ampliados, como las categorías de análisis que permiten entender la evolución y transformación de los territorios fronterizos tradicionales.

[2] Esta denominación hace referencia a los Grandes Proyectos de los años 60' y 70'.

[3] Denominaciones como "Capitales del MERCOSUR" "MERCO ciudades", etc., son ya frecuentes.

[4] Fronteras que al posicionarse desde un país en un mercado ampliado adquieren características de "internas" al mercado y/o "externas" al mismo en los bordes.

[5] Especialmente movimientos al interior del país y desde las fronteras, atrayendo poblaciones de zonas expulsoras.

[6] "Cicatrices de la historia"...

[7] Se utiliza la denominación de "par de frontera" para las ciudades gemelas en espacios fronterizos que comparten relaciones tanto funcionales como sociales y culturales, intercambio de bienes y servicios. Otras condiciones favorables son las de contar con núcleos urbanos a ambos lados de las fronteras, que en este trabajo son considerados como "Par de Frontera" y ser además lugares de paso obligado para el transporte internacional de cargas y pasajeros. Un ejemplo de ese modelo es el caso de las ciudades par de frontera como Paso de los libresUruguaiana, en la frontera entre Brasil y Argentina.

[8] En este sentido sería interesante analizar los efectos generados por la construcción del puente ResistenciaCorrientes, el puerto de Barranqueras, el corredor transcontinental bioceánico con estas dos ciudades como nudos articuladores y otras obras y proyectos, en relación al discurso oficial y menos oficial de los actores promotores de los mismos. 